\title{
CaGrid Global Model Exchange
}

National Cancer Institute

\section{Source}

National Cancer Institute. caGrid Global Model Exchange. NCI Thesaurus. Code C74931.

A grid service that provides a repository responsible for storing and linking data models as defined within namespaces in a distributed environment. It enables other services to publish, retrieve, discover, remove, and version metadata definitions. 